\title{
IMPROVED TECHNOLOGY OF CARROT CULTIVATION UNDER DRIP IRRIGATION
}

\author{
Yu.N. Pleskachev, O.G. Chamurliev, L.V. Gubina \\ Volgograd State Agricultural University \\ Volgograd, 400002, Russian Federation \\ pleskachiov@yandex.ru
}

\begin{abstract}
The article considers technologies of carrot cultivation of Shantene Korolevskaya hybrid under irrigation conditions on light chestnut soils of the Lower Volga region. Against the background of various methods of tillage, modern methods of using water-soluble fertilizers have been studied. It was found that deep chisel plowing with $0.18-0.20 \mathrm{~m}$ soil overturning results in 1.25 fold increase in productivity of edible carrot compared to moldboard plowing and blade cultivation. Using water-soluble fertilizer NS 30:7 through fertigation increases productivity by $7 \%$ compared with ammonium nitrate. Measurements of soil density showed that carrot yield averaged $1.25-1.32 \mathrm{t} / \mathrm{m}^{3}$ over $2015-2017$. In variants after deep chisel plowing rate of water infiltration during all three research years was the highest and averaged $4.2 \mathrm{~mm} / \mathrm{min}$. The smallest water infiltration was observed in variants after blade cultivation. Using ammonia nitrate during fertigation in 1-4 applications and NS 30:7 fertilizer in 5-8 applications, and NS 30:7 fertilizer during all 8 applications increased carrot yield of hybrid Shantene Korolevskaya by 4.7-5.9 and $0.5-2.4 \mathrm{t} / \mathrm{ha}$ compared to control. In addition, combination of deep chisel plowing with ammonium nitrate fertigation in 1-4 applications and NS 30:7 fertilizer in 5-8 applications resulted in the highest carrot yield in 2015-2017 and averaged 90.6 t/ha. The lowest carrot yield over 2015-2017 was observed in the variant after blade cultivation with ammonium nitrate fertigation and amounted to $77.5 \mathrm{t} / \mathrm{ha}$.
\end{abstract}

Key words: carrots, drip irrigation, tillage, fertigation

\section{INTRODUCTION}

Growing vegetables, both in protected and open ground, is rising to a new quality level now. Hence, agricultural science has to develop modern technologies based on the use of high-productive equipment and progressive irrigation methods, such as combined, fine and sprinkler $[1,2]$.

Carrots, like other vegetables, respond well to the use of high-quality seeds - first generation hybrids, innovative irrigation techniques, water-soluble complex fertilizers and effective methods of deep tillage [3-6].

The high aridity of the territory with light chestnut soils requires obligatory watering of vegetable crops during the entire growing season [7].

The main reason for fertigation popularity is the effective absorption of nutrients from irrigation water. The introduction of fertigation, as an innovative way to use watersoluble fertilizers, can significantly reduce the production costs of their placing. Moreover, synchronizing water and nutrients supply to the roots of vegetable crops leads to 
a significant increase in the efficiency of fertilizer use. Despite the overall positive result, fertigation requires the use of modern complex water-soluble fertilizers, which can increase potential productivity of vegetables and significantly improve economic indicators of their production [8].

Therefore, the use of complex water-soluble fertilizers through fertigation in carrot cultivation leads to a significant cost reduction and formation of high yields [9, 10].

\section{MATERIALS AND METHODS}

According to the results of previous field experiments we found optimum irrigation regime for carrot, which was adapted to soil and weather conditions of the light-brown soils subzone. The regime had $80 \%$ of FMC differentiated pre-irrigation soil moisture threshold in $0.2 \mathrm{~m}$ soil layer during period 'seeding — beginning of root formation', $90 \%$ of FMC moisture threshold in $0.4 \mathrm{~m}$ soil layer during period 'beginning of root formation - technical maturity' and $75 \%$ of FMC during period 'technical maturity full maturity'.

Two-factor experiments including variants of deep tillage and variants of fertigation were conducted in accordance with generally accepted methods and included in factor A three variants of deep tillage: moldboard plowing at $0.25 \ldots 0.27 \mathrm{~m}$, blade cultivation at $0.25 \ldots 0.27 \mathrm{~m}$ and chisel plowing at $0.38 \ldots 0.40 \mathrm{~m}$ with soil overturning at $0.18 \ldots$ $0.20 \mathrm{~m}$. Factor B involved three variants of water-soluble fertilizers applied: ammonium nitrate; ammonium nitrate at $1-4$ applications and NS 30:7 fertilizer in 5-8 applications; NS 30:7 fertilizer nutrient solution.

Doses of fertilizers were calculated based on projected yield of $80 \mathrm{t} / \mathrm{ha}$ and amounted to: nitrogen — $210 \mathrm{~kg} / \mathrm{ha}$, phosphorus — $130 \mathrm{~kg} / \mathrm{ha}$ and potassium $110 \mathrm{~kg} / \mathrm{ha}$. Fertilizers were added to soil in the following way: $50 \%$ of nitrogen, $100 \%$ of phosphorus and $100 \%$ of potassium were added during deep tillage, and $50 \%$ of nitrogen was added as additional fertilizing during irrigation.

The polyfactorial experiment had split-plot design with four replications. Size of first order plots was $396 \mathrm{~m}^{2}(8.4 \times 45 \mathrm{~m})$, sown area of second order plots was $126 \mathrm{~m}^{2}$ $(8.4 \times 15 \mathrm{~m})$, registration area of second order plots was $64 \mathrm{~m}^{2}(6.4 \times 10 \mathrm{~m})$.

Determination of soil density was performed by the method of cutting rings on horizons $0 \ldots 10 ; 10 \ldots 20$; and $20 \ldots 30 \mathrm{~cm}$. Infiltration losses of soil moisture were measused by lysimetric method. Weed infestation was determined by the quantitativeweighing method.

The experiments were carried out at the territory of Y.Y. Lemyakin farm in the subzone of light chestnut soils. Depending on composition soils can be characterized as heavy loamy, humus content in $0 \ldots 0.3 \mathrm{~m}$ horizon is on average $1.98 \%$.

Carrots were cultivated according to regional recommendations.

Shantene Korolevskaya edible carrot hybrid was cultivated after winter rye grown as green manure crop. Seeds were sown at the rate recommended by seed producers 1 million seeds/ha. We used drip irrigation system of Israeli company Netafim. Venturi injection pump was used for fertigation. 


\section{RESULTS AND DISCUSSION}

In spring soil density was in the range of $1.25 \ldots 1.32 \mathrm{t} / \mathrm{m}^{3}$ over research years from 2015 to 2017.

During the phase of root formation soil density in plots after chisel plowing was 0.03 and $0.06 \mathrm{t} / \mathrm{m}^{3}$ smaller than in plots after other cultivation methods of deep tillage. Before harvesting this tendency remained, and topsoil density in plots after chisel plowing was smaller by $0.03 \mathrm{t} / \mathrm{m}^{3}$ compared to moldboard plowing, and $0.07 \mathrm{t} / \mathrm{m}^{3}$ smaller compared to blade cultivation.

Table 1

Topsoil density depending on soil cultivation (average for 2015-2017), t/ $\mathbf{m}^{3}$

\begin{tabular}{|l|c|c|c|}
\hline \multicolumn{1}{|c|}{ Variant } & Before seeding & Root formation & $\begin{array}{c}\text { Before har- } \\
\text { vesting }\end{array}$ \\
\hline Moldboard plowing at 0.25-0.27 m & 1.28 & 1.34 & 1.37 \\
\hline Blade cultivation at 0.25-0.27 m & 1.32 & 1.37 & 1.41 \\
\hline $\begin{array}{l}\text { Chisel plowing at 0.38-0.40 m } \\
\text { with 0.18-0.20 m soil overturning }\end{array}$ & 1.25 & 1.31 & 1.34 \\
\hline $\mathrm{LSD}_{05} 2015$ & 0.02 & 0.02 & 0.01 \\
\hline $\mathrm{LSD}_{05} 2016$ & 0.02 & 0.01 & 0.01 \\
\hline $\mathrm{LSD}_{05} 2017$ & 0.02 & 0.02 & 0.02 \\
\hline
\end{tabular}

Rate of water infiltration in variants with deep chisel plowing turned out to be the highest over the research years and was within $4.2 \mathrm{~mm} / \mathrm{min}$. The lowest water infiltration was observed in variants with blade cultivation and averaged $3.7 \mathrm{~mm} / \mathrm{min}$.

Irrigated plots under prolonged vegetable cultivation are characterized by higher weed infestation, which does not completely eliminate herbicide applications. This circumstance requires a thorough review of tillage system when growing vegetable crops, as tillage is one of the effective ways to control weeds in vegetable crop rotations.

In the experiment Stump herbicide was applied to soil before planting carrots. Deep tillage also influenced significantly weed infestation. Weed number on average for 2015-2017 in the phase of root formation ranged from 0.4 plants $/ \mathrm{m}^{2}$ after chisel plowing to 2.6 plants $/ \mathrm{m}^{2}$ after blade cultivation.

The total number of weeds increased before harvesting, and the advantage of tillage with soil overturning became more obvious. Weed vegetation in variants with blade cultivation was $2.6 \ldots 3.3$ fold higher compared to chisel plowing with soil overturning.

Table 2

Effect of tillage on weed infestation during root formation of carrot, plants $/ \mathrm{m}^{2}$

\begin{tabular}{|l|c|c|c|c|}
\hline \multicolumn{1}{|c|}{ Variant } & 2015 & 2016 & 2017 & Average \\
\hline Moldboard plowing at 0.25-0.27 m & 0.4 & 0.6 & 0.7 & 0.6 \\
\hline Blade cultivation at 0.25-0.27 m & 1.0 & 1.4 & 2.6 & 1.3 \\
\hline $\begin{array}{l}\text { Chisel plowing at 0.38-0.40 m } \\
\text { with 0.18-0.20 m soil overturning }\end{array}$ & 0.5 & 0.7 & 0.8 & 0.7 \\
\hline $\mathrm{LSD}_{05}$ & 0.08 & 0.06 & 0.08 & \\
\hline
\end{tabular}


During research years carrot yields depending on fertigation averaged $84.7 \ldots$ $90.6 \mathrm{t} /$ ha after deep chisel plowing, 81.2 .. 85.9 and $77.5 \ldots 82.4 \mathrm{t} /$ ha after moldboard plowing and blade cultivation that is $3.5 \ldots 4.7$ and $7.2-8.2 \mathrm{t} /$ ha lower compared to chisel plowing.

Table 3

Impact of tillage and fertigation methods on carrot yield, $t / h a$

\begin{tabular}{|c|c|c|c|c|c|}
\hline Tillage & Fertigation & 2015 & 2016 & 2017 & Average \\
\hline \multirow{3}{*}{$\begin{array}{l}\text { Moldboard plowing } \\
\text { at } 0.25 \ldots 0.27 \mathrm{~m}\end{array}$} & $\mathrm{NH}_{4} \mathrm{NO}_{3}$ & 79.7 & 80.5 & 83.4 & 81.2 \\
\hline & $\mathrm{NH}_{4} \mathrm{NO}_{3}+\mathrm{NS} 30: 7$ & 84.5 & 84.9 & 88.3 & 85.9 \\
\hline & NS 30:7 & 83.2 & 84.0 & 85.1 & 84.1 \\
\hline \multirow{3}{*}{$\begin{array}{l}\text { Blade cultivation } \\
\text { at } 0.25 \ldots 0.27 \mathrm{~m}\end{array}$} & $\mathrm{NH}_{4} \mathrm{NO}_{3}$ & 75.4 & 77.1 & 80.0 & 77.5 \\
\hline & $\mathrm{NH}_{4} \mathrm{NO}_{3}+\mathrm{NS} \mathrm{30:7}$ & 79.8 & 81.7 & 85.7 & 82.4 \\
\hline & NS 30:7 & 79.4 & 81.2 & 85.1 & 81.9 \\
\hline \multirow{4}{*}{$\begin{array}{l}\text { Chisel plowing } \\
\text { at } 0.38 \ldots 0.40 \mathrm{~m} \\
\text { with } 0.18 \ldots 0.20 \mathrm{~m} \text { soil } \\
\text { overturning }\end{array}$} & $\mathrm{NH}_{4} \mathrm{NO}_{3}$ & 83.1 & 84.3 & 86.7 & 84.7 \\
\hline & $\mathrm{NH}_{4} \mathrm{NO}_{3}+\mathrm{NS} 30: 7$ & 89.0 & 89.8 & 93.0 & 90.6 \\
\hline & NS $30: 7$ & 86.3 & 87.9 & 90.4 & 88.2 \\
\hline & $\mathrm{LSD}_{05} \mathrm{AB}$ & 0.06 & 0.08 & 0.08 & \\
\hline
\end{tabular}

Application of ammonia nitrate (through fertigation) in 1-4 additional fertilizations and NS 30:7 fertilizer in 5-8 additional fertilizations increased carrot yield (Shantene Korolevskaya hybrid) by $4.7 \ldots 5.9$ t/ha. Application of NS 30:7 in 8 additional fertilizations resulted in $0.5 \ldots 2.4 \mathrm{t} / \mathrm{ha}$ yield increase compared to ammonia nitrate fertigation in 2015-2017.

The highest carrot yield was observed when combined chisel plowing and ammonium nitrate fertigation in 1-4 applications and NS 30:7 fertilizing in further 5-8 applications amounted to $90.6 \mathrm{t} / \mathrm{ha}$ in $2015-2017$.

The lowest carrot yield was $77.5 \mathrm{t} / \mathrm{ha}$ after blade cultivation and ammonium nitrate fertigation on average for $2015-2017$.

\section{CONCLUSIONS}

Regarding tillage, chisel plowing at $0.38 \ldots 0.40 \mathrm{~m}$ with $0.18 \ldots 0.20 \mathrm{~m}$ soil overturning results in the highest carrot yields on light chestnut soils of Volga-Don interfluve under drip irrigation; the most effective fertigation variant was $1-4$ ammonium nitrate applications and following 5-8 NS 30:7 fertilizer applications.

\section{REFERENCES}

1. Lemyakin YY, Skorokhodov EA. Effect of soil cultivation and herbicides on carrot's yield. Agrarian science. 2007; (9):15-16. (In Russ).

2. Pleskachev YN, Gubina LV. Innovative technologies of carrot cultivation in the Volga-Don interfluves. In: Mezhdunarodnaya nauchnaya konferentsiya "Innovatsionnye tekhnologii v rastenievodstve i ekologii”, posvyashchennoi 80-letiyu professora A.T. Farnieva; Vladikavkaz, 21 February 2017. Vladikavkaz: Gorskii GAU Publ.; 2017. p. 38 - 41. (In Russ).

3. Borodychev VV, Martynova AA. Modern carrot drip irrigation technology. In: Novye napravleniya $v$ reshenii problem APK na osnove sovremennykh resursosberegayushchikh, innovatsionnykh tekhnologii. Volgograd: VGSKhA Publ; 2010. p. 327-330. (In Russ). 
4. Borodychev VV, Serdyukova TV, Martynova AA Optimal methods of carrot growing with drip irrigation provide a high yield. Potato and vegetables. 2011; (8):11-12. (In Russ).

5. Zhidkov VM, Gubina LV. Optimal water and nutrition regimes of carrot under drip irrigation. Potato and vegetables. 2012; (1): 9-10. (In Russ).

6. Pleskachev YN, Gubina LV, Es'kov ID. Methods of increasing the yield capacity of carrot in the Volga—Don interfluve area. Nauchnaya zhizn'. 2017; (4):21—27. (In Russ).

7. Vaneyan SS, Menshikh AM. Effect of micro-irrigation and fertilizers on yield and quality of edible carrot different hybrids. Melioratsiya $i$ vodnoe khozyaistvo. 2015; (3):30-32. (In Russ).

8. Gubina LV. The use of drip irrigation in carrot cultivation in the Volga-Don interfluves. In: Materialy mezhdunarodnoi nauchno-prakticheskoi konferentsii, posvyashchennoi 70-letiyu Volgogradskogo gosudarstvennogo agrarnogo universiteta i kafedry 'Zemledelie i agrokhimiya', 14 May 2014. Volgograd: VGAU Publ.; 2014. p. 465-468. (In Russ).

9. Zhidkov VM, Khripchenko AV. The effect of tillage and herbicide application on productivity of beetroot under drip irrigation. Agrarian science. 2014; (12):18-20. (In Russ).

10. Pleskachev YN, Skorokhodov EA. Effect of tillage and herbicides on carrot cultivation under irrigation in Volgograd region. Pochvovedenie i agrokhimiya. 2013; (2):32-37. (In Russ).

\title{
INFORMATION ABOUT AUTHORS
}

Yurii Nikolaevich Pleskachev - doctor of agricultural sciences, professor, Volgograd State Agricultural University, e-mail: pleskachiov@yandex.ru

Omarii Georgievich Chamurliev - doctor of agricultural sciences, professor, Volgograd State Agricultural University; e-mail: attika.ge@yandex.ru

Larisa Vladimirovna Gubina - senior lecturer, Volgograd State Agricultural University, e-mail: agro@volgau.com

\section{For citation:}

Pleskachev YN, Chamurliev OG, Gubina LV. Improving technology of carrot cultivation under drip irrigation. RUDN Journal of Agronomy and Animal Industries, 2018, 13 (4), 360-365. doi: 10.22363/2312-797X-2018-13-4-360-365.

\section{СОВЕРШЕНСТВОВАНИЕ ТЕХНОЛОГИИ ВОЗДЕЛЫВАНИЯ МОРКОВИ НА КАПЕЛЬНОМ ОРОШЕНИИ}

\author{
Ю.Н. Плескачёв, О.Г. Чамурлиев, Л.В. Губина \\ Волгоградский государственный аграрный университет \\ пр. Университетский, 26, Волгоград, 400002, Российская Федерация \\ pleskachiov@yandex.ru
}

\begin{abstract}
Рассматриваются приемы возделывания моркови столовой гибрида «Шантенэ Королевская» в условиях орошения на светло-каштановых почвах Нижнего Поволжья. На фоне различных способов обработки почвы изучались современные методы использования водорастворимых удобрений. Было установлено, что глубокое чизелевание с оборотом пласта на $0,18-0,20$ м увеличивает в 1,25 раза продуктивность моркови столовой в сравнении с менее глубокими отвальными и плоскорезными обработками. Использование водорастворимого удобрения NS 30:7 в виде фертигации повышает продуктивность в сравнении с аммиачной селитрой на 7\%. Измерения плотности почвы
\end{abstract}


показали, что в среднем за 3 года, с 2015 по 2017 гг., весной она находилась в пределах 1,251,32 т/м³. На вариантах глубокого чизельного рыхления скорость инфильтрации воды все три года исследований оказывалась наибольшей и в среднем находилась в пределах 4,2 мм/мин. Наименьшая инфильтрация воды отмечалась на вариантах плоскорезной обработки. Применение во время фертигации моркови аммиачной селитры в первые 4 подкормки и удобрения NS 30:7 в 5-8 подкормки и удобрения NS 30:7 во время всех 8 подкормок по сравнению со стандартной фертигацией аммиачной селитрой повышало урожайность моркови столовой гибрида «Шантенэ Королевская» в среднем на $4,7-5,9$ и $0,5-2,4$ т/га. Также отмечено, что при сочетании глубокого чизельного рыхления с использованием во время фертигации аммиачной селитры в первые 4 подкормки и удобрения NS 30:7 в 5-8 подкормки в среднем за 2015-2017 гг. наблюдалась наибольшая урожайность моркови столовой и равнялась 90,6 т/га. Наименьшая же урожайность моркови столовой в среднем за 2015-2017 гг. наблюдалась на варианте плоскорезной обработки с применением во время фертигации питательного раствора с аммиачной селитрой и составляла 77,5 т/га.

Ключевые слова: морковь столовая, капельное орошение, обработка почвы, фертигация

\section{БИБЛИОГРАФИЧЕСКИЙ СПИСОК}

1. Лемякин Ю.Ю., Скороходов Е.А. Воздействие обработки почвы и гербицидов на урожайность моркови // Аграрная наука. 2007. № 9. С. 15-16.

2. Плескачев Ю.Н., Губина Л.В. Инновационные технологии возделывания моркови в ВолгоДонском междуречье // Материалы Международной научной конференции «Инновационные технологии в растениеводстве и экологии», посвященной 80-летию профессора А.Т. Фарниева, 21 февраля 2017 г. Владикавказ: Горский ГАУ, 2017. С. $38-41$.

3. Бородычев B.B., Мартынова А.А. Современная технология капельного орошения моркови // Новые направления в решении проблем АПК на основе современных ресурсосберегающих, инновационных технологий. Волгоград, 2010. Т. 1. С. 327-330.

4. Бородычев В.В., Сердюкова Т.В., Мартынова А.А. Оптимальные приемы возделывания моркови при капельном орошении обеспечивает высокий урожай // Картофель и овощи. 2011. № 8. С. 11-12.

5. Жидков В.М., Губина Л.В. Оптимальные водный и пищевой режимы выращивания моркови при капельном орошении // Картофель и овощи. 2012. № 1. С. 9-10.

6. Плескачев Ю.Н., Губина Л.В., Еськов И.Д. Приемы повышения урожайности моркови в условиях Волго-Донского междуречья // Научная жизнь. 2017. № 4. С. 21-27.

7. Ванеян С.С., Меньших А.М. Влияние микроорошения и минеральных удобрений на урожайность и качество моркови столовой разных гибридов // Мелиорация и водное хозяйство. 2015. № 3. C. $30-32$.

8. Губина Л.В. Применение капельного полива при возделывании моркови в условиях ВолгоДонского междуречья // Материалы международной научно-практической конференции, посвященной 70-летию Волгоградского государственного аграрного университета и кафедры «Земледелие и агрохимия» (14 мая 2014 г). Волгоград: Волгоградский ГАУ, 2014. C. $465-468$.

9. Жидков В.М., Хрипченко А.В. Влияние обработки почвы и внесения гербицидов на урожайность столовой свеклы при капельном орошении // Аграрная наука. 2014. № 12. C. $18-20$.

10. Плескачев Ю.Н., Скороходов Е.А. Эффективность использования обработки почвы и гербицидов при выращивании моркови на орошаемых землях Волгоградской области // Почвоведение и агрохимия. 2013. № 2. С. 32-37.

\section{Для цитирования:}

Плескачёв Ю.Н., Чамурлиев О.Г., Губина Л.В. Совершенствование технологии возделывания моркови на капельном орошении // Вестник Российского университета дружбы народов. Серия: Агрономия и животноводство. 2018. Т. 13. № 4. С. 360 -365. doi: 10.22363/2312-797Х-201813-4-360-365. 